\title{
Results and perspectives on hadron physics at KLOE/KLOE-2
}

\author{
V. De Leo ${ }^{a, \dagger, *}$ \\ aUniversità degli Studi di Roma,"La Sapienza" \\ Piazzale Aldo Moro, Roma, Italy
}

E-mail: vdeleo@roma1.infn.it

The KLOE-2 data-taking at the $e^{+} e^{-} \mathrm{DA} \Phi$ NE collider in Frascati has been completed by achieving an integrated luminosity of more than $5 \mathrm{fb}^{-1}$ at the $\phi$ peak. KLOE-2 is an updated version of the KLOE experiment with new detectors and an extended physics program, including light mesons investigations at unprecedented statistics. The KLOE/KLOE-2 data sample corresponds to more than $3 \times 10^{8}$ eta-meson events. The huge available statistics has been used to search for the $\mathrm{P}$, CP violating $\eta \rightarrow \pi^{+} \pi^{-}$, obtaining the most stringent upper limit for this decay by using $1.7 \mathrm{fb}^{-1}$ of KLOE data. The status of the $\eta \rightarrow \pi^{0} \gamma \gamma$ decay will be reported and also the progress on the investigation on the B boson, a postulated leptophobic mediator of dark forces, which uses the same five photon final state. Among the aims of the KLOE-2 experiment there is the study of the reaction $e^{+} e^{-} \rightarrow e^{+} e^{-} \gamma \gamma \rightarrow e^{+} e^{-} X$ by tagging the final state of the leptons. Data stability studies, based on very low angle Bhabha cross section measurement, and updates on $\gamma \gamma \rightarrow \pi^{0}$ analysis will be presented.

40th International Conference on High Energy physics - ICHEP2020

July 28 - August 6, 2020

Prague, Czech Republic (virtual meeting)

\footnotetext{
*Speaker

${ }^{\dagger}$ on behalf of the KLOE-2 Collaboration
} 


\section{Introduction}

The KLOE experiment has collected $2.5 \mathrm{fb}^{-1}$ of data at the peak of the phi-meson mass ( 1020 $\mathrm{MeV}$ ), plus $250 \mathrm{pb}^{-1}$ off-peak ( $\left.1000 \mathrm{MeV}\right)$, starting from 2001 to 2006 , at the $e^{+} e^{-}$collider DAФNE in Frascati. In 2008, to increase the number of collisions that the beams can produce, the Crab-Waist collision scheme has been implemented together with a large beam crossing angle[1]. In November 2014, a new data-taking campaign, referred to as KLOE-2 experiment, has started and has been concluded in March 2018 after collecting a data set of $5.5 \mathrm{fb}^{-1}$. The KLOE detector[2] consisted of a large cylindrical drift chamber (DC), surrounded by lead-scintillating fibers electromagnetic calorimeter (EMC) inserted in a $0.52 \mathrm{~T}$ magnetic field. Different upgrades to the KLOE detector have been applied in occasion of the KLOE-2 run. The Inner Tracker has been installed between the beam pipe and the inner wall of the DC to increase the acceptance for low transverse momentum tracks and improve charged vertex reconstruction[3]. New calorimeters has been added to enhance the photon detection efficiency: two identical Crystal CAlorimeter with Timing (CCALT), very close to the interaction point (IP), to increase the acceptance for particles coming from the IP from $20^{\circ}$ to $10^{\circ}$ [4] and two new Quadrupole CALorimeter with Tiles (QCALT) located around the DAФNE low- $\beta$ quadrupoles[4]. A tagging system composed of two different detectors has been installed, the Low Energy Tagger (LET) inside the KLOE detector at $1 \mathrm{~m}$ from the IP and the High Energy Tagger (HET) at $11 \mathrm{~m}$ from the IP for measuring the deviations of electrons and positrons with respect the main orbit[5].

In this paper recent results on hadron physics obtained with the KLOE data are reported (Sec. 2,3,4) while the $\gamma \gamma$ studies ongoing at KLOE-2 are discussed in the Sec.5.

\section{Upper limit on the $\eta \rightarrow \pi^{+} \pi^{-}$branching fraction}

In the Standard Model (SM) the P and CP violating decay $\eta \rightarrow \pi^{+} \pi^{-}$can proceed only through $\mathrm{CP}$ violating weak interactions via a virtual $K_{S}^{0}$ meson, with an expected branching fraction less than $2 \times 10^{-27}$. The introduction of a possible CP-violating term in the QCD lagrangian would raise this limit at $\sim 3 \times 10^{-17}$. A branching fraction of $1.2 \times 10^{-15}$ is foreseen allowing additional $\mathrm{CP}$ violation phases in the extended Higgs sector. The observation of a branching fraction larger than the above mentioned would be a signal of new sources of $\mathrm{CP}$ violation and could be useful to clarify the origin of the observed baryon asymmetry in the Universe (BAU)[2]. The best upper limit (UL) on this branching ratio has been published by the KLOE collaboration analyzing a data set of 350 $\mathrm{pb}^{-1}, \operatorname{Br}\left(\eta \rightarrow \pi^{+} \pi^{-}\right)<1.3 \times 10^{-5}$ at $90 \%$ confidence level (CL)[6]. Recently a new limit has been set also by the LHCb Collaboration by using a statistics of $\sim 3.0 \mathrm{fb}^{-1}, \operatorname{Br}\left(\eta \rightarrow \pi^{+} \pi^{-}\right)<1.6 \times 10^{-5}$ at 90\% confidence level (CL)[7]. A new analysis has been performed by the KLOE Collaboration on the search of the $\eta \rightarrow \pi^{+} \pi^{-}$decay based on an integrated luminosity of $1.61 \mathrm{fb}^{-1}$, of data collected in years 2004 and 2005[2].

To identify the $\phi \rightarrow \eta \gamma$ with $\eta \rightarrow \pi^{+} \pi^{-}$events, a photon and two tracks of opposite curvature are required. The two tracks are emitted at large polar angle values, $45^{\circ}<\theta<135^{\circ}$. The recoil photon candidate is selected by requiring an isolated energy cluster in the EMC not associated to any track. To discharge the background from $e^{+} e^{-} \rightarrow \pi^{+} \pi^{-} \gamma$ process, the angle between the direction of the missing momentum of the two tracks and the direction of the recoil photon is demanded to be 
less than $0.05 \mathrm{rad}$. The other background channels are given by $e^{+} e^{-} \rightarrow e^{+} e^{-} \gamma, e^{+} e^{-} \rightarrow \mu^{+} \mu^{-} \gamma$, $\phi \rightarrow \rho^{ \pm} \pi^{\mp}$ with $\rho^{ \pm} \rightarrow \pi^{ \pm} \gamma$, and $\phi \rightarrow \pi^{+} \pi^{-} \pi^{0}$ with an undetected photon. Time of flight (TOF) information has been used to discriminate $\pi^{+} \pi^{-} \gamma$ and $e^{+} e^{-} \gamma$ events. To separate $\mu^{+} \mu^{-} \gamma$ and $\pi^{+} \pi^{-} \pi^{0}$ events a cut on the track mass variable $\left(M_{T R K}\right)$ has been applied; it was computed by assuming the $\phi$ decays to two identical mass particles and a photon. Events survived to these selection criteria have been used to search for the decay $\eta \rightarrow \pi^{+} \pi^{-}$. The $\pi^{+} \pi^{-}$invariant mass spectrum, $M_{\pi^{+} \pi^{-}}$, in the $\eta$ mass region $[500,600] \mathrm{MeV} / \mathrm{c}^{2}$, is shown as black dots in Fig. 1. The irreducible background in $\eta$ signal region $[540,555] \mathrm{MeV} / \mathrm{c}^{2}$ is evaluated by performing a fit to the $\eta$ sidebands, [500,540] and $[555,600] \mathrm{MeV} / \mathrm{c}^{2}$, with a third-order polynomial function. The $\eta$ signal is described by the

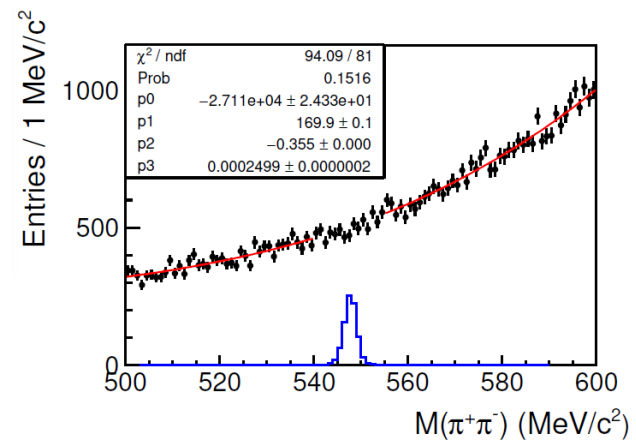

Figure 1: $\pi^{+} \pi^{-}$invariant mass distribution; the dots with error bars are data, the red lines represent the fit result to $\eta$ sidebands and the blue histogram is the $\eta$ signal with the branching fraction arbitrarily fixed for visualisation[2]

corresponding MC simulated shape, the blue histogram in Fig. 1; signal branching fraction has been arbitrarily fixed to $8.8 \times 10^{-5}$ just for visualization purposes. Since there is no evident peak observed in the $\eta$ mass region, an upper limit on the branching fraction of $\eta \rightarrow \pi^{+} \pi^{-}$is extracted with the CLs technique and resulted: $\operatorname{Br}\left(\eta \rightarrow \pi^{+} \pi^{-}\right)<4.9 \times 10^{-6}$ at $90 \% \mathrm{CL}$. This sample has been combined with the one of the previous KLOE analysis[6] giving: $\mathrm{Br}\left(\eta \rightarrow \pi^{+} \pi^{-}\right)<4.4 \times 10^{-6}$. Considering instead the full KLOE-KLOE-2 statistics the expected UL on the branching fraction is: $\operatorname{Br}\left(\eta \rightarrow \pi^{+} \pi^{-}\right)<2.7 \times 10^{-6}$ at $90 \% \mathrm{CL}[2]$.

\section{3. $\eta \rightarrow \pi^{0} \gamma \gamma$ analysis}

The $\eta \rightarrow \pi^{0} \gamma \gamma$ decay is an important test of chiral perturbation theory (ChPT) because of its sensitivity to the $p^{6}$ term on both the branching ratio and the $\mathrm{M}(\gamma \gamma)$ spectrum. The published measurements on the branching fraction of the process are given from CrystalBall@AGS[8] and CrystalBall@MAMI[9]. An old preliminary analysis has been performed also by the KLOE Collaboration by using a sample of $450 \mathrm{pb}^{-1}$ and the following preliminary result has been obtained: $\operatorname{Br}\left(\eta \rightarrow \pi^{0} \gamma \gamma\right)=\left(8.4 \pm 2.7_{\text {stat }} \pm 1.4_{\text {syst }}\right) \times 10^{-5}[10]$.

A new KLOE analysis is in progress using a statistics that is almost 4 times higher than the previous one, $\sim 1.7 \mathrm{fb}^{-1}$. The reaction studied is the $\phi \rightarrow \eta \gamma$ decay looking to the final state with five prompt photons. A prompt photon is an EMC cluster with no charged tracks associated and that 
satisfies the requirement: $|t-r / c|<\min \left[5 \sigma_{t}(E), 2 \mathrm{~ns}\right]$ where $t$ corresponds to the photon time of flight, $r$ is path length, and $c$ is the speed of light. In the Fig. 2 (left) the preliminary invariant mass
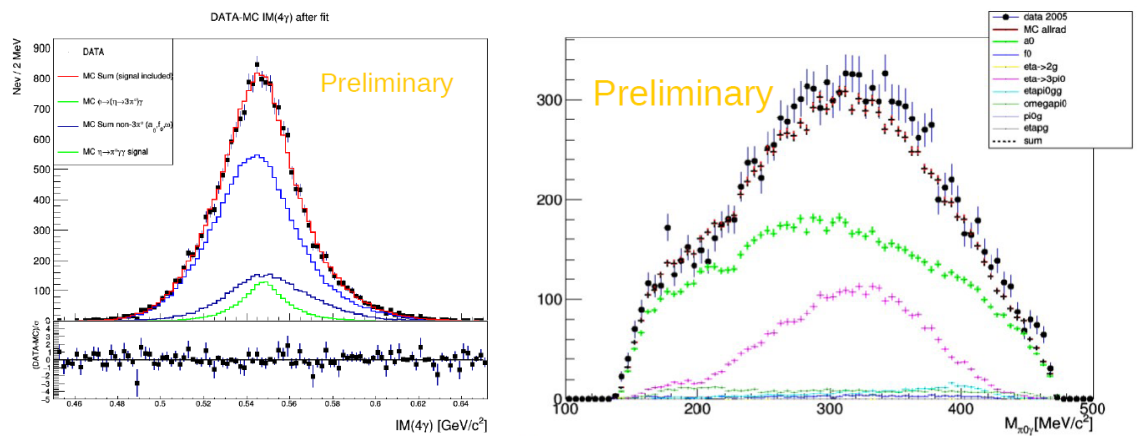

Figure 2: (Left) $4 \gamma$ invariant mass spectrum: black points refer to the data, the red line corresponds to the MC prediction that includes the sum of the background and signal events, the light blue line to the background from the $\eta \rightarrow 3 \pi^{0}$ events, the dark blue line to the other background channels and the green line to the MC signal. In the down part the difference between data and MC is reported. (Right) Invariant Mass distribution of the $\pi^{0} \gamma$ events: solid dots correspond to data while the crosses refer to the Monte Carlo simulated processes.

spectrum of the $4 \gamma$ is reported: black points are referred to the data, the red line corresponds to the MC prediction that includes the sum of background and signal events, the green one to the MC signal shape while the other distributions are referred to the background composition, namely, the $\eta \rightarrow 3 \pi^{0}$ decay with lost or merged photons (light blue histogram) and the other background reactions coming from the $\phi \rightarrow \gamma a_{0}, \phi \rightarrow \gamma f_{0}$ and $\phi \rightarrow \pi^{0} \omega$ (dark blue histogram). The background rejection has been performed by means of kinematic fit with energy and momentum conservation constraints. A multivariate analysis (TMVA-BDT) has been also implemented in order to reduce the big amount of background due to the $\eta \rightarrow 3 \pi^{0}$ events ( $\sim 50 \%$ of rejected events).

\section{B boson search}

New gauge symmetries may exist beyond the SM and light meson decay are very suitable for discovering new forces below the $\mathrm{GeV}$ scale. The existence of a leptophobic mediator between the dark sector and the SM particles, the B boson, has been hypothesized. Although the B boson couples predominantly to quarks, it may be observed in rare radiative decays of $\eta, \eta^{\prime}, \omega, \phi$ mesons as a $\pi^{0} \gamma$ resonance[11]. The study of the $\phi \rightarrow \eta \gamma$ process with B that decays in $\pi^{0} \gamma$ is the object of the KLOE search. The analyzed sample corresponds to an integrated luminosity of $\sim 1.7 \mathrm{fb}^{-1}$. As in the above described analysis, five prompt photons are selected. The main background comes from the $\phi \rightarrow \gamma a_{0}\left(\eta \pi^{0}\right)$ and $\phi \rightarrow \gamma \eta\left(3 \pi^{0}\right)$ which can mimic five photon events due to photon loss or cluster merging. Kinematic fits are performed in order to suppress background imposing the energy and total 4-momentum conservation and the speed of light for each photon. In case of evidence of the B-boson, it should appear in the invariant mass distribution of the $\pi^{0} \gamma$ events; preliminary results are shown in the Fig. 2 (right) where solid dots correspond to data while the crosses refer to the Monte Carlo simulated processes. No signal signature was found. 


\section{5. $\gamma \gamma$ physics at KLOE-2}

Scintillator hodoscopes installed by means of roman pots in the DA $\Phi$ NE beam pipe allow to investigate $\gamma \gamma$ physics at the $\phi$ resonance from the reaction $e^{+} e^{-} \rightarrow e^{+} e^{-} \gamma \gamma \rightarrow e^{+} e^{-} X$ by tagging final state leptons[12]. The analysis for the measurement of the width $\Gamma_{\pi^{0} \rightarrow \gamma \gamma}$ is in progress, aiming to achieve an accuracy of $\mathrm{O}(1 \%)$. The $\pi^{0}$ production from two-photon fusion is tagged by requiring the coincidence between the hits in one HET station and 2 clusters in the KLOE detector originating from the same bunch crossing $\left(A_{+}\right.$sample), and evaluating the uncorrelated HET-KLOE time coincidences (A sample). The HET counting rate resulted dominated by low-angle radiative Bhabha scattering. The evaluation of the cross section of this process is very useful to check the HET detector operational stability and validate acceptance and efficiency of the detector by comparison with the BBBREM simulation[13].
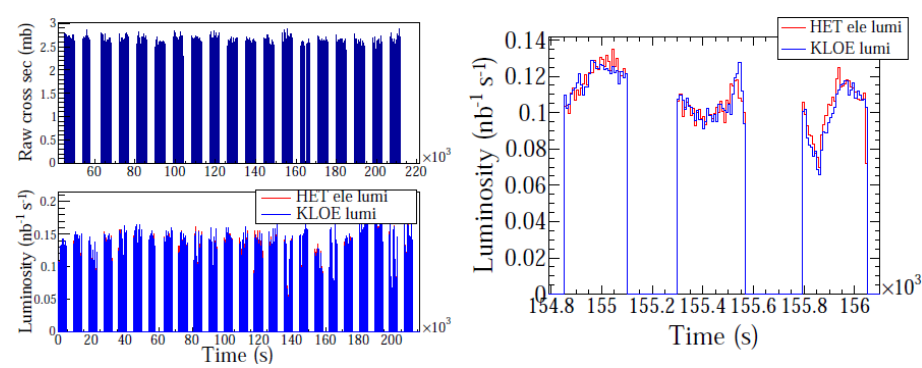

Figure 3: (Left upper) Measurement of the low angle raw-radiative Bhabha cross section $\left(\sigma_{B H A}\right)$ per second performed by the HET electron station. (Left down) Luminosity patterns as measured by KLOE and by the HET. (Right) Zoom of the luminosity pattern.

The stability of the raw radiative $\sigma_{B H A}$ measured by the HET electron station has been verified. The results are shown in the Fig. 3 (left upper) together with the luminosity patterns as evaluated by KLOE and by the HET electron station (left down). A zoom of the luminosity pattern is reported on the right part of the Fig. 3 where it's possible to see the agreement between the two different measurements from the HET and KLOE detectors[13]. The comparison of $A / A_{+}$samples for $1 \mathrm{fb}^{-1}$
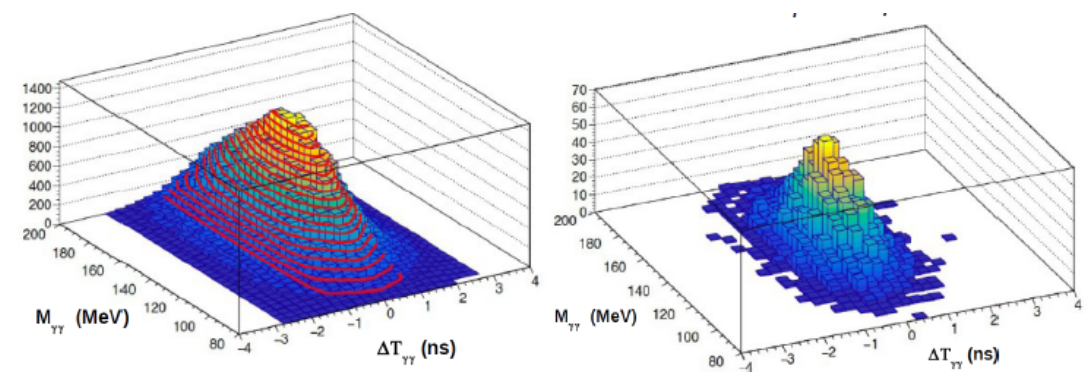

Figure 4: (Left) 2D-fit of the $A_{+}$sample with 2 Gaussian components, signal and accidentals. (Right) 2D-fit of the $A_{+}-A$ data sample to estimate the tagged events.

data set shows $3.5(0.7) \mathrm{k}$ tagged events in the mass region where $\pi^{0}$ from fusion are expected (Fig. 4). To improve the precision of the measurement, a new signal modelling that profits of detailed 
study on resolution and trigger threshold has been finalized and a multivariate analysis is ongoing on the full reconstructed data sample $\left(\sim 2.5 \mathrm{fb}^{-1}\right)$.

\section{Conclusions}

The KLOE/KLOE-2 data sample corresponds to $2.4 \times 10^{10} \phi$ mesons. The large data sample of light mesons available provided important results on decay dynamics together with limits on new physics: the most stringent UL on the $\eta \rightarrow \pi^{+} \pi^{-}$decay has been presented and also the preliminary results on the B boson search, $\eta \rightarrow \pi^{0} \gamma \gamma$ decay and the progress on the $\pi^{0}$ search.

\section{References}

[1] C. Milardi et al., High luminosity interaction region design for collisions inside high field detector solenoid, JINST 7 (2012) T03002.

[2] D. Babusci et al., Upper limit on the $\eta \rightarrow \pi^{+} \pi^{-}$branching fraction with the KLOE experiment, JHEP 10 (2020) 047.

[3] A. Di Cicco et al., Commissioning of the Inner Tracker of the KLOE-2 experiment, Acta Phys. Pol. B 46 (2015) 73.

[4] F. Happacher et al., Commissioning of the New Calorimeters of the KLOE-2 Experiment, Acta Phys. Pol. B 46 (2015) 87.

[5] D. Babusci et al., Commissioning of the new taggers of the KLOE-2 experiment, Acta Phys. Pol. B 46 (2015) 81.

[6] F. Ambrosino et al., Upper limit on the $\eta \rightarrow \pi^{+} \pi^{-}$branching ratio with the KLOE detector, PLB 606 (2005) 276.

[7] R. Aaij et al., Search for the CP-violating strong decays $\eta \rightarrow \pi^{+} \pi^{-}$and $\eta^{\prime}(958) \rightarrow \pi^{+} \pi^{-}$, PLB 764 (2017) 233.

[8] S. Prakhov et al., Measurement of the invariant-mass spectrum for the two photons from the $\eta \rightarrow \pi^{0} \gamma \gamma$ decay, PRC 78 (2008) 015206.

[9] B. M. K. Nefkens et al., New measurement of the rare decay $\eta \rightarrow \pi^{0} \gamma \gamma$ with the Crystal Ball/TAPS detectors at the Mainz Microtron, PRC 90 (2014) 025206.

[10] B. Di Micco et al., The $\eta \rightarrow \pi^{0} \gamma \gamma$ decay, the $\eta / \eta^{\prime}$ mixing angle and the $\eta$ mass measurement at KLOE, Acta Phys. Slov. 56 (2006) 403.

[11] Tulin et al., New weakly coupled forces hidden in low-energy QCD, Phys. Rev. D 89 (2014) 114008 .

[12] D. Babusci et al., On the possibility to measure the $\pi^{0} \rightarrow \gamma \gamma$ decay width and the $\gamma^{*} \gamma \rightarrow \pi^{0}$ transition form factor with the KLOE-2 experiment, EPJ C 72 (2012) 1917.

[13] F. Curciarello, The KLOE-2 $e^{+} e^{-}$tagging for two-photon physics, JINST 15 (2020) C09016. 